\title{
Effectiveness of Superficial Radial Nerve Block on Pain, Function and Quality of Life in Patients With Hand Osteoarthritis: A Prospective, Randomized and Controlled Single-Blind Study
}

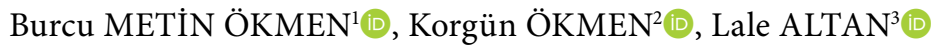 \\ ${ }^{1}$ Department of Physical Medicine and Rehabilitation, University of Health Sciences, Bursa Yüksek İhtisas Training and Research Hospital, Bursa, Turkey \\ ${ }^{2}$ Department of Anesthesiology and Reanimation, University of Health Sciences, Bursa Yüksek İhtisas Training and Research Hospital, Bursa, Turkey \\ ${ }^{3}$ Department of Physical Medicine and Rehabilitation, Uludă̆ University Faculty of Medicine, Bursa, Turkey
}

\begin{abstract}
Objectives: This study aims to investigate the effects of ultrasound-guided superficial branch of the radial nerve (SBRN) block on pain, function and quality of life in patients with hand osteoarthritis (HOA).

Patients and methods: In this prospective, randomized and controlled single-blind study, 50 female patients (mean age $59.0 \pm 5.1$ years; range, 46 to 64 years) diagnosed with HOA were randomized into two groups. Group $1(n=25)$ was administered an ultrasound-guided SBRN injection (with a combination of $2 \mathrm{~mL} 0.25 \%$ bupivacaine and $1 \mathrm{~mL}$ methylprednisolone acetate) + exercise, while group $2(\mathrm{n}=25)$ only exercised. Patients were assessed with a Visual Analog Scale for pain, hand grip strength (HGS), finger grip strength (FGS), Quick Disability of the Arm, Shoulder and Hand questionnaire, Duruöz Hand Index and Short-form 12. Data were obtained before treatment (W0), in the second week after treatment (W2) and in the fourth week after treatment (W4).

Results: In group 1, a statistically significant improvement was obtained in all parameters at both W2 and W4 compared to the values recorded at W0 ( $p<0.05$ ). In group 2, a statistically significant improvement was observed only in the parameters of HGS and FGS at W2 and W4 ( $p<0.05$ ). A comparison of the scores of the two groups showed statistically significant superior improvement in group 1 in all parameters at both W2 and W4 $(\mathrm{p}<0.05)$.

Conclusion: The findings of our study showed that an ultrasound-guided SBRN block combined with exercise is a significantly superior treatment to exercise-only regarding the improvements in the parameters of pain, function and quality of life in HOA patients.

Keywords: Exercise; hand osteoarthritis; nerve block; superficial branch of the radial nerve; ultrasound.
\end{abstract}

Osteoarthritis $(\mathrm{OA})$ is one of the most common joint disorders, ${ }^{1}$ and hand osteoarthritis (HOA) is the most frequent form of $\mathrm{OA},{ }^{2}$ with a high and increasing prevalence that poses a substantial burden on patients and community. ${ }^{3}$ Clinical manifestations of HOA are soft-tissue swelling and bony enlargements, most frequently in the distal and proximal interphalangeal finger joints and in the trapeziometacarpal (TM) joint of the thumb, ${ }^{4}$ with subsequent limitations in daily activities due to pain, stiffness and reduced range of motion $(\mathrm{ROM})^{5}$ in the joints, as well as a decrease of over $60 \%$ in grip strength. ${ }^{6}$

The basic goal of HOA treatment is to control the symptoms through pharmacological and nonpharmacological agents, ${ }^{7,8}$ since, to our knowledge, there is currently no cure for HOA. ${ }^{7}$ Pharmacological modalities are usually limited to symptomatic treatment, while patients with severe thumb TM OA are generally referred to surgery

Received: November 25, 2017 Accepted: February 01, 2018 Published online: March 23, 2018

Correspondence: Burcu Metin Ökmen, MD. Sağlık Bilimleri Üniversitesi, Bursa Yüksek İhtisas Eğitim ve Araştırma Hastanesi, Fiziksel Tıp ve Rehabilitasyon Kliniği, 16310 Yıldırım, Bursa, Turkey. Tel: +90 224 - 2955000 e-mail: burcumetinokmen@gmail.com 
when conservative interventions fail to suppress the symptoms. The European League Against Rheumatism (EULAR) states that the optimal management of HOA involves a combination of non-pharmacologic and pharmacologic treatments that should be planned in accordance with the clinical status and expectations of the patient. ${ }^{7}$ All patients should be given instruction in exercises that involve joint protection, $\mathrm{ROM}$ and strengthening. ${ }^{7}$

The superficial branch of the radial nerve (SBRN) is a purely sensory nerve that usually starts in the cubital fossa, ${ }^{9}$ which assumes a subcutaneous course of, on average, $9 \mathrm{~cm}$, proximal to the radial styloid process of the radius, and bifurcates into its terminal branches at a mean distance of $5.1 \mathrm{~cm}$ proximal to that process. ${ }^{10}$ SBRN innervates the dorsoradial metacarpal area, the dorsum of the thumb, the index and middle fingers, and dorsoradial half of the ring finger. ${ }^{9}$

Peripheral nerve blocks are currently used as a treatment modality for osteoarthritic pain, as well as for other conditions involving chronic pain. ${ }^{11-14}$ There have been several studies that evaluated the efficacy of saphenous ${ }^{12}$ and genicular blocks in knee OA, ${ }^{13}$ suprascapular nerve blocks in shoulder $\mathrm{OA},{ }^{11}$ and obturator and femoral nerve blocks in hip OA. ${ }^{14}$ In a review, it has been emphasized that the employment of injections into these nerves with the guidance of ultrasonography (US) would increase the success rate. ${ }^{15}$ US has been gaining popularity in the diagnosis and monitoring of several peripheral and central system disorders as a portable, non-invasive, radiation-free, less expensive and easily accessible tool. ${ }^{16}$ US is currently used as a guide during invasive interventions into the musculoskeletal system, with the aim being to avoid the risk of iatrogenic complications due to blind injections. ${ }^{16}$ We could not identify any published studies that evaluated the efficacy of nerve blocks in HOA. Hence, in this study, we aimed to investigate the effects of US-guided SBRN block on pain, function and quality of life in the patients with HOA.

\section{PATIENTS AND METHODS}

In this prospective, randomized and controlled single-blind study, 60 patients with the diagnosis of HOA according to American College of Rheumatology criteria ${ }^{17}$ were evaluated for eligibility at University of Health Sciences, Bursa Yüksek İhtisas Training and Research Hospital between January 2017 and June 2017. The inclusion criteria were: (i) to be between the ages of 35 and 65 , (ii) to have sufficient intellectual capacity to understand the content of the clinical assessment scales, (iii) to have experienced hand pain for more than three months that involved at least two hand joints, and (iv) to have a diagnosis of HOA compatible with a modified Kellgren and Lawrence grade of $\geq 2$. The exclusion criteria were: (i) to have been diagnosed with diabetes mellitus, (ii) to have been diagnosed with cervical radiculopathy, (iii) to have been diagnosed with polyneuropathy, (iv) to have been diagnosed with inflammatory rheumatic disease, $(v)$ to have been diagnosed with carpal tunnel syndrome, (vi) to have been diagnosed with malignancy, (vii) to have a history of surgical intervention in the neck area, (viii) patients with certain surgical indications, (ix) to have a history of hand surgery, $(x)$ to have uncontrolled hypertension, (xi) to have a history of physical therapy and/or injections for HOA within the past six months, or (xii) to be undergoing anticoagulant therapy.

A total of 50 female patients (mean age $59.0 \pm 5.1$ years; range, 46 to 64 years) were deemed eligible for the study. Patients' demographic characteristics and pre-treatment (W0) measurements were recorded. The study protocol was approved by the University of Health Sciences, Bursa Yüksek İhtisas Training and Research Hospital Ethics Committee (decision number: 2011-KAEK-25 2016/21-03). A written informed consent was obtained from each patient. The study was conducted in accordance with the principles of the Declaration of Helsinki.

Patients were divided into two groups using a random number table. Group $1(n=25)$ was treated with US (Esaote MyLab30, Genoa, Italy)guided SBRN injection+exercise, while group 2 $(n=25)$ only exercised (Figure 1).

For US-guided SBRN block, patients were seated with the elbow at a $90^{\circ}$ flexion and the dorsoradial hand portion exposed. The skin was cleaned, and then, SBRN was identified by placing the $10-15 \mathrm{MHz}$ Linear US probe at a point $10 \mathrm{~cm}$ proximal to the radial styloid in the 


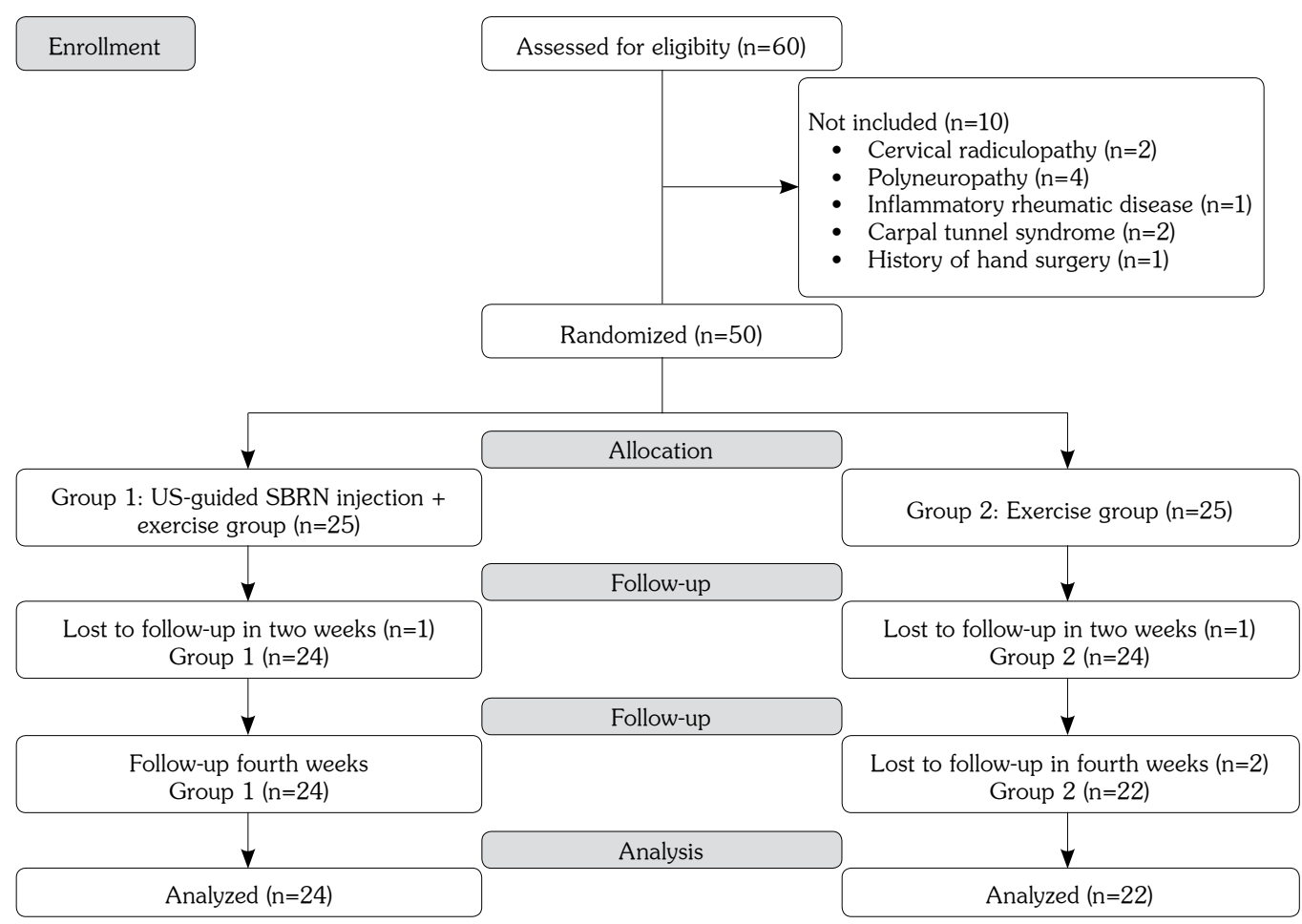

Figure 1. Flow chart of this study. US: Ultrasonography; SBRN: Superficial branch of the radial nerve.

sagittal position. Next, a combination of $2 \mathrm{~mL}$ $0.25 \%$ bupivacaine and $1 \mathrm{~mL}$ methylprednisolone acetate was applied around the nerve using the inplane technique with a $5 \mathrm{~cm} 22$-gauge US-visible needle. The needle was then removed, and a sterile pressure dressing and cold pack were placed over the injection site (Figure 2).

Regarding the exercise treatment, all patients were instructed about ROM and strengthening exercises and the dose was adjusted to avoid provoking pain by an experienced physiotherapist not related to the study. The exercises, which included small fist, large fist, tabletop, okay signs, finger spread, thumb reach, gripping, key pinch and fingertip pinch, ${ }^{18}$ were performed at home once a day with 10 repeats in each session. Patients were strictly advised to perform the exercises regularly and inquired as to their adherence to the protocol during the control visits.

The same researcher, who was blind to the type of treatment, completed post-treatment second
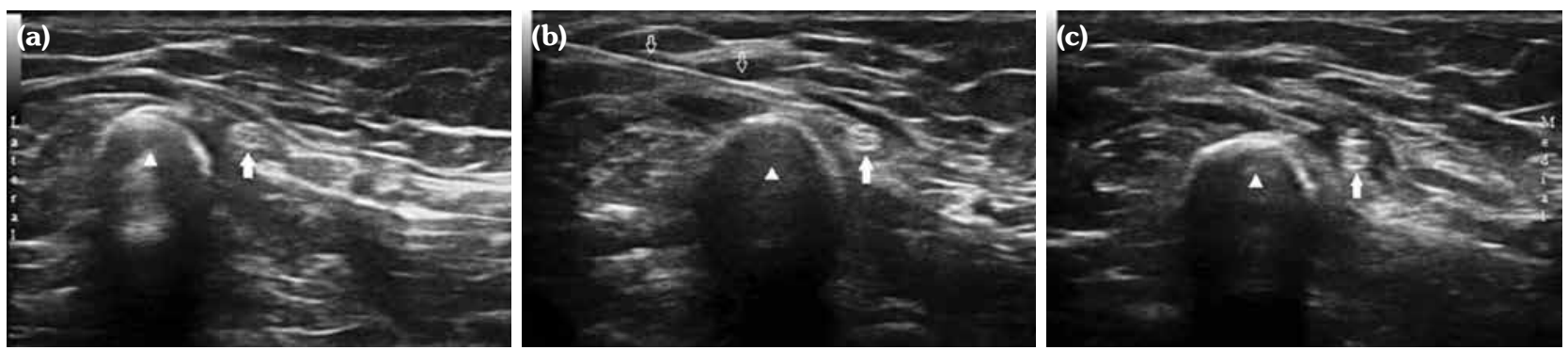

Figure 2. Superficial branch of radial nerve block. (a) Superficial radial nerve. (b) Needle and superficial radial nerve during injection. (c) Superficial radial nerve (white arrow) surrounded by hypoechoic (local anesthetic) area after local anesthetic application. Triangle shows bone of radius. White arrow shows superficial radial nerve. Empty arrows show needle. 
Table 1. Comparison of demographic characteristics of patients

\begin{tabular}{|c|c|c|c|c|c|c|c|}
\hline & \multicolumn{3}{|c|}{ Group $1(\mathrm{n}=24)$} & \multicolumn{3}{|c|}{ Group $2(\mathrm{n}=22)$} & \multirow[b]{2}{*}{$p$} \\
\hline & $\mathrm{n}$ & $\%$ & Mean $\pm \mathrm{SD}$ & $\mathrm{n}$ & $\%$ & Mean $\pm \mathrm{SD}$ & \\
\hline Age (year) & & & $58.8 \pm 5.5$ & & & $59.2 \pm 4.8$ & 0.821 \\
\hline Body Mass Index $\left(\mathrm{kg} / \mathrm{m}^{2}\right)$ & & & $29.3 \pm 2.9$ & & & $30.3 \pm 4.0$ & 0.325 \\
\hline \multicolumn{8}{|l|}{ Sex } \\
\hline Females & 24 & 100 & & 22 & 100 & & NA \\
\hline Affected hand & & & & & & & 0.801 \\
\hline Right & 14 & 58.3 & & 12 & 54.5 & & \\
\hline Left & 10 & 41.7 & & 10 & 44.5 & & \\
\hline Complaint duration (month) & & & $24.2 \pm 23.1$ & & & $21.1 \pm 14.5$ & 0.596 \\
\hline
\end{tabular}

week (W2) and fourth week (W4) evaluations and recorded the data.

Visual Analog Scale was used to assess the severity of the pain felt by the patient. ${ }^{19}$ The standard Jamar Dynamometer (Jamar $^{\circledR}$ Plus+ Digital Hand D ynamometer from Patterson Medical by Sammons Preston, Illinois, USA) was used to measure hand grip strength (HGS). The validity and reliability of the Jamar Dynamometer are high, and it is regarded as the gold standard in the assessment of grip strength. ${ }^{20}$ The measurements were performed bilaterally three times in the seated position, with the shoulder in adduction, the elbow at $90^{\circ}$ flexions, and the forearm between supination and pronation. The mean values of results were recorded in kilograms.

Table 2. Comparison of week 0 , week 2 and week 4 values within and between groups

\begin{tabular}{|c|c|c|c|c|c|c|c|c|}
\hline & \multicolumn{2}{|r|}{ W0 } & \multicolumn{2}{|r|}{ W2 } & \multicolumn{2}{|r|}{ W4 } & \multirow{2}{*}{$\frac{(\mathrm{W} 2-\mathrm{W} 0)}{p}$} & \multirow{2}{*}{$\frac{(\mathrm{W} 4-\mathrm{W} 0)}{p}$} \\
\hline & Median & Min-Max & Median & Min-Max & Median & Min-Max & & \\
\hline \multicolumn{9}{|l|}{ Visual Analog Scale } \\
\hline Group $1(n=24)$ & 8 & $6-9$ & 4 & $2-6$ & 1 & $0-6$ & $<0.001$ & $<0.001$ \\
\hline Group $2(\mathrm{n}=22)$ & 8 & $5-9$ & 8 & $5-9$ & 8 & 5-9 & 0.851 & 0.539 \\
\hline$p$ & \multicolumn{2}{|r|}{0.729} & \multicolumn{2}{|r|}{$<0.001$} & \multicolumn{2}{|r|}{$<0.001$} & & \\
\hline \multicolumn{9}{|l|}{ DASH } \\
\hline Group $1(\mathrm{n}=24)$ & 41.67 & $22.50-80.00$ & 26.67 & $4.17-54.17$ & 16.67 & $2.50-41.67$ & $<0.001$ & $<0.001$ \\
\hline Group 2 ( $n=22)$ & 50.83 & $14.17-62.50$ & 50.83 & $14.17-62.50$ & 51.25 & $19.17-62.50$ & 0.107 & 0.304 \\
\hline$p$ & \multicolumn{2}{|c|}{0.826} & \multicolumn{2}{|r|}{0.031} & \multicolumn{2}{|r|}{$<0.001$} & & \\
\hline \multicolumn{9}{|l|}{ DHI } \\
\hline Group $1(n=24)$ & 22.5 & $7-63$ & 16.5 & $2-50$ & 9.5 & $1-25$ & $<0.001$ & $<0.001$ \\
\hline Group $2(\mathrm{n}=22)$ & 32 & $7-57$ & 31 & 7-59 & 29.5 & 8-57 & 0.873 & 0.982 \\
\hline$p$ & \multicolumn{2}{|r|}{0.234} & \multicolumn{2}{|r|}{0.008} & \multicolumn{2}{|r|}{$<0.001$} & & \\
\hline \multicolumn{9}{|l|}{ HGS } \\
\hline Group $1(n=24)$ & 36.10 & $12.10-46.30$ & 41.10 & $21.30-53.50$ & 45.9 & $27.60-58.70$ & $<0.001$ & $<0.001$ \\
\hline Group 2 (n=22) & 35.00 & $19.70-49.00$ & 35.75 & $22.80-50.10$ & 37.7 & $25.30-51.20$ & $<0.001$ & $<0.001$ \\
\hline$p$ & \multicolumn{2}{|c|}{0.843} & \multicolumn{2}{|c|}{0.286} & \multicolumn{2}{|r|}{0.009} & & \\
\hline \multicolumn{9}{|l|}{ FGS } \\
\hline Group 1 (n=24) & 7.5 & $4-12$ & 9 & $7-14$ & 11 & $9-17$ & $<0.001$ & $<0.001$ \\
\hline Group 2 (n=22) & 8 & 5-13 & 9 & 6-14 & 11 & $7-15$ & $<0.001$ & $<0.001$ \\
\hline$p$ & \multicolumn{2}{|r|}{0.392} & \multicolumn{2}{|r|}{0.679} & \multicolumn{2}{|r|}{0.056} & & \\
\hline \multicolumn{9}{|c|}{ Short-form 12} \\
\hline \multicolumn{9}{|c|}{ Physical component summary } \\
\hline Group $1(n=24)$ & 36.65 & $22.60-44.70$ & 40.85 & $30.10-45.00$ & 46.10 & $32.70-49.10$ & $<0.001$ & $<0.001$ \\
\hline Group $2(\mathrm{n}=22)$ & 35.50 & $25.50-43.30$ & 33.50 & $24.10-42.10$ & 36.50 & $25.50-41.80$ & 0.431 & 0.575 \\
\hline$p$ & \multicolumn{2}{|r|}{0.826} & \multicolumn{2}{|c|}{$<0.001$} & & $<0.001$ & & \\
\hline Mental component s & & & & & & & & \\
\hline Group $1(n=24)$ & 34.65 & $30.2-43.3$ & 39.45 & $36.6-42.5$ & 42.00 & $39.60-48.20$ & $<0.001$ & $<0.001$ \\
\hline Group $2(\mathrm{n}=22)$ & 35.90 & $20.3-50.4$ & 37.10 & $22.30-49.20$ & 35.9 & $21.3-46.3$ & 0.295 & 0.851 \\
\hline$p$ & & 0.217 & & 0.113 & & 0.006 & & \\
\hline
\end{tabular}


Finger grip strength (FGS) was measured using a pinch gauge (Jamar ${ }^{\circledR}$ Pinch gauge, Illinois, USA) three times bilaterally by asking the patients to squeeze the gauge with maximal power using the tips of the thumb and the index finger, and the results were recorded in kilograms. The validity and reliability of the FGS were tested by Mathiowetz et al. ${ }^{21}$

Quick Disability of the Arm, Shoulder and Hand questionnaire (DASH) is a validated and widely used 11-item questionnaire that measures specific symptoms and disabilities in the upper extremities ( 0 no disability, 100 the most serious disability). One advantage of the Quick DASH is that it can be used to assess any region of the upper extremities. ${ }^{22}$

Duruöz Hand Index (DHI) contains 18 items related to hand ability in the kitchen, during dressing, while performing personal hygiene, while performing office tasks, among other general items. Persons rate their ability from 0 (no difficulty) to 5 (impossible to do). The questionnaire yields a score from 0 to 90 and takes about three minutes to complete. DHI has been found to be reliable and valid in patients with OA. ${ }^{23}$

In short-form 12 (SF-12), 12 different items are taken from eight different headings of SF-36. SF-12 has physical (SF-12-physical component summary) and mental (SF-12-mental component summary) state assessment scales, of which regression analyses have been performed on the general public. The physical and mental health sum scales are computed using the scores of 12 questions and range from 0 to 100 , where a zero score indicates the lowest level of health measured by the scales, and 100 indicates the highest level of health. ${ }^{24}$

No drugs (e.g. analgesics, non-steroid antiinflammatory drugs, gabapentin, pregabalin) were administered to patients throughout this study.

Table 3. Comparison of difference between scores of groups

\begin{tabular}{|c|c|c|c|c|}
\hline & \multicolumn{2}{|c|}{ W2-W0 } & \multicolumn{2}{|r|}{ W4-W0 } \\
\hline & Median & Min-Max & Median & Min-Max \\
\hline \multicolumn{5}{|l|}{ Visual Analog Scale } \\
\hline Group $1(\mathrm{n}=24)$ & -4 & $-6 ;-2$ & -6.5 & $-8 ;-2$ \\
\hline Group $2(\mathrm{n}=22)$ & 0 & $-1 ; 3$ & 0 & $-1 ; 2$ \\
\hline$p$ & \multicolumn{2}{|c|}{$<0.001$} & \multicolumn{2}{|r|}{$<0.001$} \\
\hline \multicolumn{5}{|l|}{ DASH } \\
\hline Group $1(\mathrm{n}=24)$ & -15.01 & $28.33 ;-8.33$ & -24.17 & $-50.83 ;-10.00$ \\
\hline Group $2(\mathrm{n}=22)$ & 0 & $-3.34 ; 2.50$ & -1.26 & $-5 ; 5.83$ \\
\hline$p$ & \multicolumn{2}{|c|}{$<0.001$} & \multicolumn{2}{|r|}{$<0.001$} \\
\hline \multicolumn{5}{|l|}{ DHI } \\
\hline Group 1 (n=24) & -7 & $-17 ;-1$ & -14 & $-38 ;-2$ \\
\hline Group $2(\mathrm{n}=22)$ & 0 & $-2 ; 3$ & 0 & $-4 ; 5$ \\
\hline$p$ & \multicolumn{2}{|c|}{$<0.001$} & \multicolumn{2}{|r|}{$<0.001$} \\
\hline \multicolumn{5}{|l|}{ HGS } \\
\hline Group $1(\mathrm{n}=24)$ & 4.65 & $-4.10 ; 18.5$ & 10.90 & $2.60 ; 26.70$ \\
\hline Group $2(\mathrm{n}=22)$ & 1.8 & $0.20 ; 3.80$ & 3.8 & $0.90 ; 6.40$ \\
\hline$p$ & \multicolumn{2}{|c|}{$<0.001$} & \multicolumn{2}{|r|}{$<0.001$} \\
\hline \multicolumn{5}{|l|}{ FGS } \\
\hline Group $1(\mathrm{n}=24)$ & 2 & $-1 ; 3$ & 4 & $1 ; 7$ \\
\hline Group $2(\mathrm{n}=22)$ & 1 & $0 ; 2$ & 2 & $1 ; 4$ \\
\hline$p$ & \multicolumn{2}{|c|}{0.001} & \multicolumn{2}{|r|}{$<0.001$} \\
\hline \multicolumn{5}{|c|}{ Short-form 12} \\
\hline \multicolumn{5}{|c|}{ Physical component summary } \\
\hline Group $1(\mathrm{n}=24)$ & 3.5 & $-1.4 ; 13.6$ & 8 & $1.2 ; 19.3$ \\
\hline Group $2(\mathrm{n}=22)$ & -1.4 & $-4.20 ; 3.30$ & -0.4 & $-2.5 ; 4.60$ \\
\hline$p$ & \multicolumn{2}{|c|}{$<0.001$} & \multicolumn{2}{|r|}{$<0.001$} \\
\hline \multicolumn{5}{|c|}{ Mental component summary } \\
\hline Group 1 (n=24) & 5.3 & $-4.20 ; 7.60$ & 7.95 & $-2.7 ; 13.1$ \\
\hline Group $2(\mathrm{n}=22)$ & 0 & $-3 ; 5.40$ & -1 & $-4.9 ; 5.40$ \\
\hline$p$ & \multicolumn{2}{|c|}{0.001} & \multicolumn{2}{|r|}{$<0.001$} \\
\hline
\end{tabular}

Min: Minimum; Max: Maximum; W: Week; DASH: Disability of the Arm, Shoulder and Hand; DHI: Duruöz Hand Index; HGS: Hand Grip Strength; FGS: Finger Grip Strength; Group 1: Ultrasonography-guided superficial radial nerve block + exercise group; Group 2: Exercise-only group. 


\section{Statistical analysis}

An analysis of the collected data was carried out using the IBM SPSS version 22.0 (IBM Corp., Armonk, NY, USA) statistical package program. A Pearson chi-square test $\left(\chi^{2}\right)$ or Yates $\chi^{2}$ test was used in the comparison of the categorical variables. The normal distribution of the data was tested using the Shapiro-Wilk test. When found to be normally distributed, an independent samples t-test was used for between-group comparisons, and when distributed abnormally, a Mann-Whitney $\mathrm{U}$ test was used for between-group comparisons and a Wilcoxon test for intra-group comparisons. Values with a probability of (p) $\alpha<0.05$ were accepted as significant.

\section{RESULTS}

One patient from group 1 (US-guided SBRN injection+exercise) and three patients from group 2 (exercise-only) withdrew from this study for private reasons. No patient was excluded from this study due to side effects of the treatment. The present study was completed with a total of 46 patients (group $1 ; n=24$ and group 2; $\mathrm{n}=22$ ) (Figure 1).

The distributions of age, sex, Body Mass Index, affected hand and complaint durations of the patients are presented in Table 1 . The pretreatment evaluations of the parameters in both groups are presented in Table 2. No statistically significant difference was identified between the two groups regarding demographic characteristics or pre-treatment evaluation parameters ( $p>0.05$ ).

In group 1 , a statistically significant improvement was obtained in all parameters, both at W2 and W4, when compared to the W0 values $(p<0.05)$ (Table 2$)$. In group 2 , significant improvement was observed only in the parameters of HGS and FGS at W2 and W4 $(p<0.05)$ (Table 2). A comparison of the difference between the scores of the two groups showed significantly superior improvements in group 1 in all parameters at both W2 and W4 $(p<0.05)$ (Table 3).

\section{DISCUSSION}

Hand $\mathrm{OA}$ is a significant health problem that may limit patients' daily activities due to pain, stiffness and reduced ROM of the joints. ${ }^{5}$ To our knowledge, this is the first study that investigated the effects of US-guided SBRN block on pain, function and quality of life in the patients with HOA. The results of this study have shown that US-guided SBRN block, combined with exercise, is significantly superior to exercise-only in improving the parameters of pain, function and quality of life both at two and four weeks after treatment in patients with HOA.

Pain is one of the most common symptoms of osteoarthritis and known to be associated with local degenerative changes, such as destruction of the cartilage, synovial inflammation and alteration of the bone. ${ }^{25,26}$ However, pain severity is not always proportional to the extent of the inflammation, and joint destruction and sensitization may also aggravate the pain felt by patients. ${ }^{27}$ Osteoarthritic pain is related to the central sensitization at the level of the cortex and spinal cord, as well as the peripheral mechanisms. ${ }^{28,29}$ Both the peripheral and central sensory pathways play a role in triggering and maintaining the mechanisms that lead to resistant pain. ${ }^{30}$ Nerve blocks are frequently used to treat chronic pain due to peripheral and central sensitization, ${ }^{31-33}$ and corticosteroid injections employed in these blocks are known to provide analgesia by blocking the transmission of nociceptive C-fibers, and also by potentiating and increasing the duration of the effects of local anesthetics in combination. ${ }^{31,32}$ Salgia et al. ${ }^{33}$ used a combination of methyl prednisolone acetate and bupivacaine, injected into the suprascapular nerve in 60 patients with chronic shoulder pain, and found out that bupivacaine reduced the sensitization of the dorsal horn neurons, while methylprednisolone acetate both increased the duration of the effects of the bupivacaine and exerted an anti-inflammatory effect.

The efficacy of nerve blocks in OA patients has been investigated in a small number of studies. ${ }^{11-14}$ Shanahan et al. ${ }^{11}$ demonstrated that injecting the suprascapular nerve with a combination of $10 \mathrm{~mL}$ of $0.5 \%$ bupivacaine and $40 \mathrm{mg}$ of methylprednisolone is a safe, effective and welltolerated treatment by patients with chronic shoulder pain that resulted from degeneration or rheumatoid arthritis, and observed significant improvements in the pain, ROM and disability of the shoulder with that regimen when compared 
to placebo. In another study on 200 patients with knee OA that had lasted for more than three months and did not respond to conservative treatment, significant improvement was seen following the injection of the saphenous nerve in the adductor canal with lidocaine and triamcinolone acetonide. Thus, the authors suggested this block as a safe and effective method in knee OA patients in whom analgesics are either contraindicated or ineffective. ${ }^{12}$

Furthermore, Choi et al. ${ }^{13}$ showed that a radiofrequency block applied to the genicular nerve is safe and effective in 38 patients who had severe knee OA for more than three months, and who had not responded to conservative treatment. In another study, blocks of the articular branches of the obturator and femoral nerves with lidocaine were found to be beneficial in reducing resistant pain in the short and mid-term in 20 patients with hip OA. ${ }^{14}$

The human thumb TM joint receives innervation from several branches, from the palmar cutaneous and thenar branches of the median nerve, the lateral cutaneous nerve of the forearm, and the SBNR terminated at or around the joint. ${ }^{34,35}$ Most patients with thumb TM OA complain about pain in the dorsum of the hand. ${ }^{36}$ We chose to use an SBRN block in this study because it vastly innervates the dorsoradial hand region, and also because it is a purely sensory nerve.

A SBRN block has been used previously in many studies. Henshaw et al. ${ }^{37}$ achieved significant pain relief using a continuous local anesthetic infusion of SBRN in a 46-year-old female with type II complex regional pain, secondary to upper extremity trauma, and suggested SBRN block as an alternative treatment method in patients with similar symptoms. An US-guided SBRN block has also been suggested in cheiralgia paresthetica that resulted from entrapment of the SBRN. ${ }^{38}$

Anatomical variations in the course and branching pattern of SBRN may cause complications during any interventions of the nerve. ${ }^{39}$ Ultrasonographic guidance is known to reduce such complications as well as technical difficulties, and to increase the success rate. ${ }^{40} \mathrm{We}$ did not experience any technical difficulties or complications when using US-guided nerve block in this study.
EULAR guidelines suggest combining both pharmacologic and non-pharmacologic modalities and the inclusion of exercise in treatment protocols for $\mathrm{HOA}^{7}$ Exercises, particularly for ROM and strengthening, are also suggested as the gold standard for OA treatment in all patients in the 2014 OA Research Society International. ${ }^{41}$ There is limited evidence of the effects of exercise on pain, ROM and strength. However, the combination of exercise with splinting has been reported to reduce pain and stiffness, and to improve function, in several reports. ${ }^{42}$ Lefler and Armstrong $^{43}$ achieved significant improvement in grip strength and ROM, but not in pain and pinch strength in the exercise group when compared to the controls in HOA patients. Rogers and Wilder ${ }^{18}$ compared exercise and sham hand cream in HOA patients, and observed significant improvements in grip and pinch strength in the exercise group yet failed to reveal any difference between the groups in hand skills and the subscales of Australian/ Canadian Hand Osteoarthritis Index.

We decided to standardize the groups in this study by employing the same exercise protocol in both groups since we thought it would be unethical to deprive the control patients of some form of treatment. As we obtained significant improvement in pinch and grip strength but not in pain and function in the exercise-only group, we may speculate that exercise may strengthen the hand musculature, but does not have any significant effect on the mechanisms related to pain and function.

The lack of a placebo intervention group and the relatively short follow-up period could be cited as obvious limitations of this study.

In conclusion, this study has shown that an US-guided SBRN block combined with exercise was significantly superior to exercise-only in the improvement of the parameters of pain, function and quality of life in HOA patients. To our knowledge, this is the first study that investigated the effects of US-guided SBRN block in HOA, and we suggest this method as a safe, convenient and effective treatment alternative in HOA patients who are unresponsive to conventional therapy in the light of the positive results of this study. We believe that further studies with placebo-controlled design and longer follow-up period with larger patient populations are required to gain a better understanding of the topic addressed in this study. 


\section{Declaration of conflicting interests}

The authors declared no conflicts of interest with respect to the authorship and/or publication of this article.

\section{Funding}

The authors received no financial support for the research and/or authorship of this article.

\section{REFERENCES}

1. Orozco L, Munar A, Soler R, Alberca M, Soler F, Huguet M, et al. Treatment of knee osteoarthritis with autologous mesenchymal stem cells: a pilot study. Transplantation 2013;95:1535-41.

2. Lawrence RC, Felson DT, Helmick CG, Arnold LM, Choi H, Deyo RA, et al. Estimates of the prevalence of arthritis and other rheumatic conditions in the United States. Part II. Arthritis Rheum 2008;58:26-35.

3. Zhang Y, Niu J, Kelly-Hayes M, Chaisson CE, Aliabadi P, Felson DT. Prevalence of symptomatic hand osteoarthritis and its impact on functional status among the elderly: The Framingham Study. Am J Epidemiol 2002;156:1021-7.

4. Botha-Scheepers S, Riyazi N, Watt I, Rosendaal FR, Slagboom E, Bellamy N, et al. Progression of hand osteoarthritis over 2 years: a clinical and radiological follow-up study. Ann Rheum Dis 2009;68:1260-4.

5. Kloppenburg M. Hand osteoarthritisnonpharmacological and pharmacological treatments. Nat Rev Rheumatol 2014;10:242-51.

6. Haugen IK, Englund M, Aliabadi P, Niu J, Clancy M, Kvien TK, et al. Prevalence, incidence and progression of hand osteoarthritis in the general population: the Framingham Osteoarthritis Study. Ann Rheum Di. 2011;70:1581-6.

7. Zhang W, Doherty M, Leeb BF, Alekseeva L, Arden NK, Bijlsma JW, et al. EULAR evidence based recommendations for the management of hand osteoarthritis: report of a Task Force of the EULAR Standing Committee for International Clinical Studies Including Therapeutics (ESCISIT). Ann Rheum Dis 2007;66:377-88.

8. Hochberg MC, Altman RD, April KT, Benkhalti M, Guyatt G, McGowan J, et al. American College of Rheumatology 2012 recommendations for the use of nonpharmacologic and pharmacologic therapies in osteoarthritis of the hand, hip, and knee. Arthritis Care Res (Hoboken) 2012;64:465-74.

9. Samarakoon LB, Lakmal KC, Thillainathan S, Bataduwaarachchi VR, Anthony DJ, Jayasekara RW. Anatomical relations of the superficial sensory branches of the radial nerve: a cadaveric study with clinical implications. Patient Saf Surg 2011;5:28.

10. Doyle JR, Botte MJ. Surgical Anatomy of the Hand and Upper Extremity. Philadelphia: Wolters Kluwer
Health; 2015.

11. Shanahan EM, Ahern M, Smith M, Wetherall M, Bresnihan B, FitzGerald O. Suprascapular nerve block (using bupivacaine and methylprednisolone acetate) in chronic shoulder pain. Ann Rheum Dis 2003;62:400-6.

12. Lee DH, Lee MY, Kwack KS, Yoon SH. Effect of adductor canal block on medial compartment knee pain in patients with knee osteoarthritis: Retrospective comparative study. Medicine (Baltimore) 2017;96:e6374.

13. Choi WJ, Hwang SJ, Song JG, Leem JG, Kang YU, Park $\mathrm{PH}$, et al. Radiofrequency treatment relieves chronic knee osteoarthritis pain: a double-blind randomized controlled trial. Pain 2011;152:481-7.

14. Yavuz F, Yasar E, Ali Taskaynatan M, Goktepe AS, Tan AK. Nerve block of articular branches of the obturator and femoral nerves for the treatment of hip joint pain. J Back Musculoskelet Rehabil 2013;26:79-83.

15. Korbe S, Udoji EN, Ness TJ, Udoji MA. Ultrasoundguided interventional procedures for chronic pain management. Pain Manag 2015;5:465-82.

16. Walker FO, Cartwright MS, Wiesler ER, Caress J. Ultrasound of nerve and muscle. Clin Neurophysiol 2004;115:495-507.

17. Altman R, Alarcón G, Appelrouth D, Bloch D, Borenstein D, Brandt K, et al. The American College of Rheumatology criteria for the classification and reporting of osteoarthritis of the hand. Arthritis Rheum 1990;33:1601-10.

18. Rogers MW, Wilder FV. Exercise and hand osteoarthritis symptomatology: a controlled crossover trial. J Hand Ther 2009;22:10-7.

19. Price DD, McGrath PA, Rafii A, Buckingham B. The validation of visual analogue scales as ratio scale measures for chronic and experimental pain. Pain 1983;17:45-56.

20. Shechtman O, Gestewitz L, Kimble C. Reliability and validity of the DynEx dynamometer. J Hand Ther 2005;18:339-47.

21. Mathiowetz V, Weber K, Volland G, Kashman N. Reliability and validity of grip and pinch strength evaluations. J Hand Surg Am 1984;9:222-6.

22. Beaton DE, Wright JG, Katz JN. Development of the QuickDASH: comparison of three itemreduction approaches. J Bone Joint Surg [Am] 2005;87:1038-46.

23. Poiraudeau S, Chevalier X, Conrozier T, Flippo RM, Lioté F, Noël E, et al. Reliability, validity, and sensitivity to change of the Cochin hand functional disability scale in hand osteoarthritis. Osteoarthritis Cartilage 2001;9:570-7.

24. Ware J Jr, Kosinski M, Keller SD. A 12-Item ShortForm Health Survey: construction of scales and preliminary tests of reliability and validity. Med Care 1996;34:220-33.

25. Felson DT. The sources of pain in knee osteoarthritis. Curr Opin Rheumatol 2005;17:624-8. 
26. Smith MD, Triantafillou S, Parker A, Youssef PP, Coleman M. Synovial membrane inflammation and cytokine production in patients with early osteoarthritis. J Rheumatol 1997;24:365-71.

27. Bradley LA, Kersh BC, DeBerry JJ, Deutsch G, Alarcón GA, McLain DA. Lessons from fibromyalgia: abnormal pain sensitivity in knee osteoarthritis. Novartis Found Symp 2004;260:258-70.

28. Im HJ, Kim JS, Li X, Kotwal N, Sumner DR, van Wijnen AJ, et al. Alteration of sensory neurons and spinal response to an experimental osteoarthritis pain model. Arthritis Rheum 2010;62:2995-3005.

29. Mease PJ, Hanna S, Frakes EP, Altman RD. Pain mechanisms in osteoarthritis: understanding the role of central pain and current approaches to its treatment. J Rheumatol 2011;38:1546-51.

30. Graven-Nielsen T, Arendt-Nielsen L. Peripheral and central sensitization in musculoskeletal pain disorders: an experimental approach. Curr Rheumatol Rep 2002;4:313-21.

31. Johansson A, Hao J, Sjölund B. Local corticosteroid application blocks transmission in normal nociceptive C-fibres. Acta Anaesthesiol Scand 1990;34:335-8.

32. Huynh TM, Marret E, Bonnet F. Combination of dexamethasone and local anaesthetic solution in peripheral nerve blocks: A meta-analysis of randomised controlled trials. Eur J Anaesthesiol 2015;32:751-8.

33. Salgia A, Agarwal T, Puri SR, Sanghi S, Mohapatra A. Role of suprascapular nerve block in chronic shoulder pain: A comparative study of 60 cases. Med J DY Patil Univ 2014;7:44-7.

34. Lorea DP, Berthe JV, De Mey A, Coessens BC, Rooze M, Foucher $\mathrm{G}$. The nerve supply of the trapeziometacarpal joint. J Hand Surg Br 2002;27:232-7.

35. Poupon M, Duteille F, Cassagnau E, Leborgne J, Pannier M. Anatomical study of the nerve supply of the trapeziometacarpal joint. Fifteen dissections. Rev Chir Orthop Reparatrice Appar Mot 2004;90:34652. [Abstract]

36. Mobargha N, Ludwig C, Ladd AL, Hagert E. Ultrastructure and innervation of thumb carpometacarpal ligaments in surgical patients with osteoarthritis. Clin Orthop Relat Res 2014;472:1146-54.

37. Henshaw DS, Kittner SL, Jaffe JD. UltrasoundGuided Continuous Superficial Radial Nerve Block for Complex Regional Pain Syndrome. J Pain Palliat Care Pharmacother 2016;30:118-23.

38. Meng S, Tinhofer I, Weninger WJ, Grisold W. Anatomical and ultrasound correlation of the superficial branch of the radial nerve. Muscle Nerve 2014;50:939-42.

39. Herma T, Baca V, Yershov D, Kachlik D. A case of a duplicated superficial branch of radial nerve and a two-bellied brachioradialis muscle presenting a potential entrapment syndrome. Surg Radiol Anat 2017;39:451-4

40. Lewis SR, Price A, Walker KJ, McGrattan K, Smith AF. Ultrasound guidance for upper and lower limb blocks. Cochrane Database Syst Rev 2015;9:CD006459.

41. McAlindon TE, Bannuru RR, Sullivan MC, Arden NK, Berenbaum F, Bierma-Zeinstra SM, et al. OARSI guidelines for the non-surgical management of knee osteoarthritis. Osteoarthritis Cartilage 2014;22:363-88.

42. Kjeken I, Smedslund G, Moe RH, SlatkowskyChristensen B, Uhlig T, Hagen KB. Systematic review of design and effects of splints and exercise programs in hand osteoarthritis. Arthritis Care Res (Hoboken) 2011;63:834-48

43. Lefler C, Armstrong J. Exercise in the treatment of osteoarthritis in the hands of the elderly. Clin Kinesiol 2004;58:13-7. 